\title{
Determination of the Binding Sites of Activators within the Proteasome Structure ${ }^{+}$
}

\author{
Malgorzata Gizynska 1,*, Julia Witkowska ${ }^{1}$, Przemyslaw Karpowicz 2, Fabian Henneberg ${ }^{3}$, \\ Ashwin Chari ${ }^{3}$, Artur Gieldon ${ }^{4}$ and Elzbieta Jankowska ${ }^{1}$ \\ 1 Department of Biomedical Chemistry, Faculty of Chemistry, University of Gdansk, 80-308 Gdańsk, Poland; \\ julia.witkowska@ug.edu.pl (J.W.) \\ 2 Department of Organic Chemistry, Faculty of Chemistry, University of Gdansk, 80-308 Gdańsk, Poland; \\ przemyslaw.karpowicz@ug.edu.pl \\ 3 Department for Structural Dynamics, Max-Planck Institute for Biophysical Chemistry, 37077 Göttingen; \\ fabian.henneberg@mpibpc.mpg.de (F.H.); ashwin.chari@mpibpc.mpg.de (A.C.) \\ 4 Department of Theoretical Chemistry, Faculty of Chemistry, University of Gdansk, 80-308 Gdańsk, Poland; \\ artur.gieldon@ug.edu.pl \\ * Correspondence: malgorzata.gizynska@phdstud.ug.edu.pl \\ + Presented at the 2nd Molecules Medicinal Chemistry Symposium (MMCS): Facing Novel Challenges in \\ Drug Discovery, Barcelona, Spain, 15-17 May 2019.
}

Published: 12 November 2019

The proteasome degrade most of the proteins in eukaryotic cells, thereby controlling the key cellular processes. Impaired degradation mechanisms can lead to accumulation of damaged proteins, resulting in the development of aging processes. Reduced activity of the proteasome also underlies the etiology of some neurodegenerative disorders, such as Alzheimer's or Parkinson's disease. It is believed that low-molecular mass proteasome activators could prevent progression of age-related neurodegenerative disorders.

Blm10 is an activator of the yeast 20S proteasome, which stimulates hydrolysis of peptides and some partially unstructured proteins. The crystal structure of the Blm10-yeast $20 \mathrm{~S}$ complex revealed that C-terminal residues of Blm10 insert into the pocket between the $\alpha 5$ and $\alpha 6$ subunits of the $20 \mathrm{~S}$ core particle, and their binding allows to partially open the gate that leads to the catalytic chamber of the proteasome [1].

Blm-pep is a 14-mer peptide, designed based on the sequence of the Blm10 protein, which efficiently stimulates chymotrypsin-like activity of human $20 S$ proteasome. The crystal structure of the complex of Blm-pep and yeast $20 S$ proteasome shows that Blm-pep docks into the same pocket as the C-terminus of Blm10 [2]. So far, we have obtained dozens of analogs of Blm-pep, which stimulate the proteasome peptidases at $10 \mu \mathrm{M}$ concentration several times. Some of these compounds also are able to enhance the rate of protein substrates degradation in vitro. To determine the place of binding of obtained activators in the structure of human proteasome, we applied different techniques such as cross-linking in combination with mass spectrometry, X-ray crystallography, and molecular modelling. These studies have revealed that there are several sites that are able to bind modulators.

Acknowledgments: This study was financially supported by NCN-funded grant 2014/15/B/NZ7/01014 and project PROM POWR.03.03.00-00-PN13/18

\section{References}

1. Sadre-Bazzaz, K.; Whitby, F.G.; Robinson, H.; Formosa, T.; Hill, C.P. Structure of a Blm10 complex reveals common mechanisms for proteasome binding and gate opening. Mol. Cell 2010, 37, 728-735 
2. Witkowska, J.; Giżyńska, M.; Grudnik, P.; Golik, P.; Karpowicz, P.; Giełdoń, A.; Dubin, G.; Jankowska, E. Crystal structure of a low molecular weight activator Blm-pep with yeast $20 \mathrm{~S}$ proteasome - insights into the enzyme activation mechanism. Sci. Rep. 2017, 7, 6177

(C) 2019 by the authors. Licensee MDPI, Basel, Switzerland. This article is an open access article distributed under the terms and conditions of the Creative Commons Attribution (CC BY) license (http://creativecommons.org/licenses/by/4.0/). 